Commentary

\title{
Cloud Computing in Singapore: Key Drivers and Recommendations for a Smart Nation
}

\author{
Reuben $\mathrm{Ng}{ }^{1,2}$ \\ ${ }^{1}$ Lee Kuan Yew School of Public Policy, National University of Singapore, 259772 Singapore, Singapore; \\ E-Mail: spprng@nus.edu.sg \\ ${ }^{2}$ Geriatric Education and Research Institute, 769027 Singapore, Singapore
}

Submitted: 1 October 2018 | Published: 21 November 2018

\begin{abstract}
Cloud computing adoption enables big data applications in governance and policy. Singapore's adoption of cloud computing is propelled by five key drivers: (1) public demand for and satisfaction with e-government services; (2) focus on whole-of-government policies and practices; (3) restructuring of technology agencies to integrate strategy and implementation; (4) building the Smart Nation Platform; (5) purpose-driven cloud applications especially in healthcare. This commentary also provides recommendations to propel big data applications in public policy and management: (a) technologically, embrace cloud analytics, and explore "fog computing" - an emerging technology that enables on-site data sense-making before transmission to the cloud; (b) promote regulatory sandboxes to experiment with policies that proactively manage novel technologies and business models that may radically change society; (c) on the collaboration front, establish unconventional partnerships to co-innovate on challenges like the skills-gap-an example is the unprecedented partnership led by the Lee Kuan Yew School of Public Policy with the government, private sector and unions.
\end{abstract}

\section{Keywords}

Keywords: big data; cloud computing; public management; psychomics; public policy; Singapore; smart city

\section{Issue}

This commentary is part of the issue "Big Data Applications in Governance and Policy", edited by Sarah Giest (Leiden University, The Netherlands) and Reuben Ng (National University of Singapore, Singapore).

(C) 2018 by the author; licensee Cogitatio (Lisbon, Portugal). This article is licensed under a Creative Commons Attribution 4.0 International License (CC BY).

\section{Introduction}

The world generates more data every two days than from the dawn of early civilization through the year 2003 combined. And data rates are still growing at approximately $40 \%$ per year. Against this background, data has outstripped common warehousing and analytics tools. To move forward, organisations-both public and privateneed new capabilities, especially cloud computing, to differentiate their products and services.

Every major study hails the potential of cloud computing and analytics. Gartner found that enterprise spending on cloud computing grew faster than overall IT spending and predicted the technology will grow by over $100 \%$ (PRWeb, 2012). Synergy Research Group concluded that in 2016, cloud computing dominated many components within the Information and Communica- tion Technologies (ICT) market, pushing cloud revenue growth above $25 \%$ year-on-year. A cloud-enabled business model survey found that $62 \%$ of Chief Information Officers and Chief Data Officers consider cloud computing as the top priority for ICT (Berman, Kesterson-Townes, Marshall, \& Srivatbsa, 2011). Bessemer Venture Partners reported that the cloud computing market revenue grew $35.8 \%$ annually from $2008-2014$, and $22.8 \%$ annually from 2014 to 2018 culminating in projected revenues of US\$127 billion (Figure 1). A 2010 survey found that $23 \%$ of Singapore companies adopted cloud technology. More recently, International Data Corporation (IDC) forecasted that the cloud computing market in Singapore will grow to US\$1 billion by the end of 2018.

There are three features of cloud computing: first, cloud computing provides services on demand, and these resources are scalable over multiple data centres. 


\section{CLOUD COMPUTING MARKET REVENUE}

2008-2018

(Figures in USD Billion)

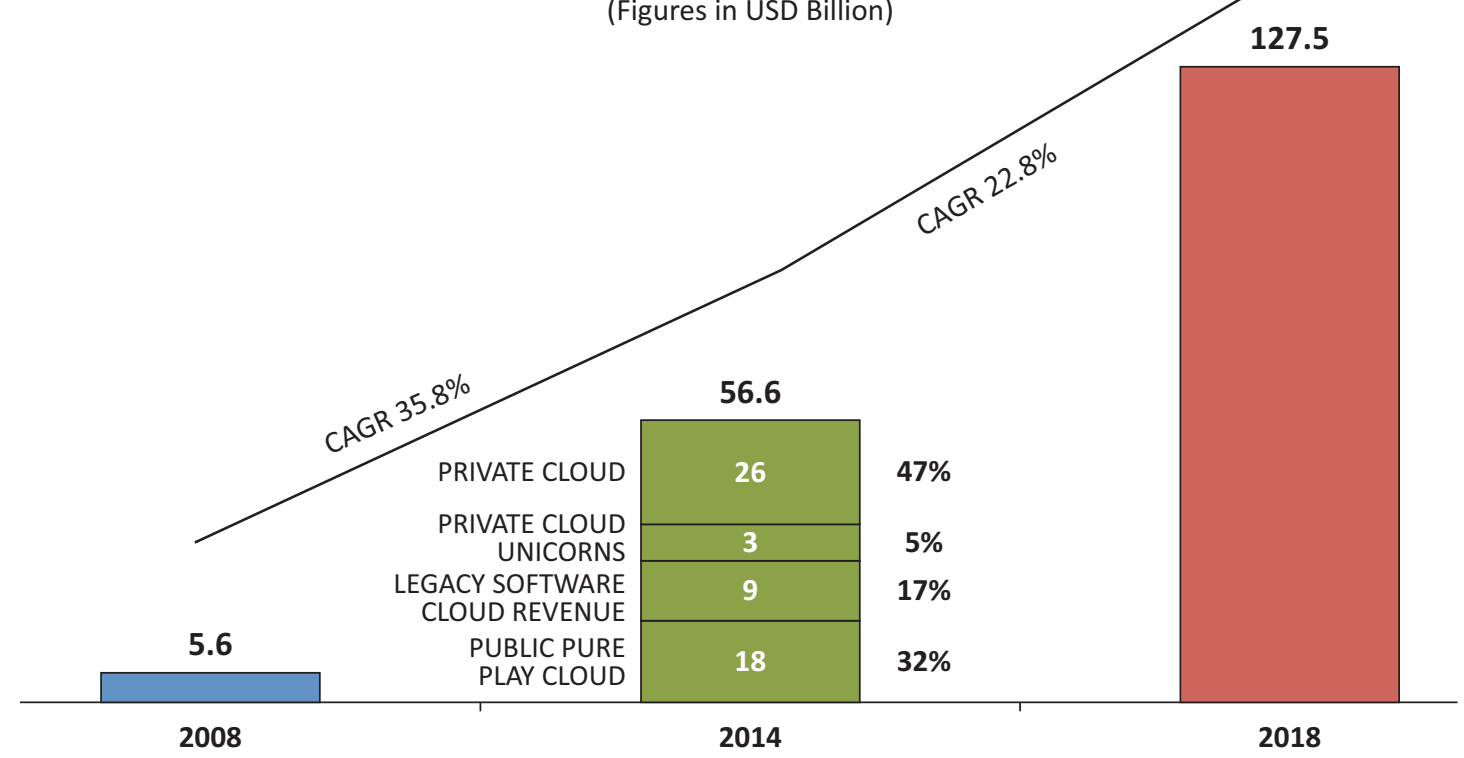

Figure 1. Incredible growth of cloud computing market revenue over a decade (2008-2018; Deeter \& Shen, 2015).

Second, these services are easily accessible and location independent, meaning that a user can access the suite of services worldwide through internet browsers. Third, service quality is guaranteed for bandwidth and memory capacity.

These features give rise to three benefits: first, cloud computing provides a more palatable cost structure. It requires no upfront hardware cost compared with traditional data warehouses with high capital investments. The use of cloud computing is recorded as operating expenses and significantly reduced. Second, business risk is reduced as it is outsourced to providers who are better equipped to manage these risks. Third, organisations who are starting their digital journey can leverage on the latest computing technology to leap frog their capabilities compared to a traditional step-wise build up.

Of broader significance, cloud computing is positioned as a crucial enabler in Singapore's Smart Nation plan. The latter is part of a national effort to put Singapore at the forefront of technological innovation and implementation. The Prime Minister took a personal interest in launching the vision in 2014 and in 2017, devoted a significant part of his National Day Rally Speech, an important annual address, to update on the nation's initiatives and aspirations.

The case study will first outline the key drivers for Singapore's adoption of cloud computing. Second, analyse the cybersecurity considerations in cloud computing adoptions. Third, provide recommendations for countries embarking on a journey to enable big data applications in public management and policy.

\section{Five Key Drivers of Singapore's Cloud Computing Adoption}

\subsection{Public Demand for and Satisfaction with E-Government Services}

Singapore has done well in ICT infrastructure development as evidenced by the extraordinary high mobile subscription penetration rate of $155.6 \%$ and, high internet penetration rate of $135.1 \%$ in 2013 . In addition, $73 \%$ of the Singapore population are internet users, exposed to the information deluge on the world wide web. The statistic is comparable to the average internet users of above $70 \%$ in developed countries. On the contrary, fixed telephone line usage has plunged to $36.4 \%$ and $25.7 \%$ for wired broadband subscriptions. These numbers underscore the high levels of connectivity and mobility of residents in Singapore.

On the public front, through e-government initiatives, Singapore migrated many processes that required faceto-face interactions to self-help channels, along with noteworthy adoption of paperless transactions. As a result, Singapore has been ranked highly in e-government implementation (Table 1), and in 2013, emerged tops ahead of Finland and the US. These initiatives have resonated with the citizenry, garnering positive feedback from 2011 to 2015 from both businesses and individuals, according to 2016 surveys from the Singapore Government Technology Agency (GovTech; Figure 2).

On the government-to-business front, over 1500 representatives from multiple industry sectors surveyed reported that $99 \%$ of businesses visited government websites and over $90 \%$ were satisfied with the information quality provided, and the ease of completing transactions online. 
Table 1. Singapore tops the ranks for e-government implementation in 2013 (Hashemi, Monfaredi, \& Masdari, 2013).

\begin{tabular}{lll}
\hline Rank & Country & Score \\
\hline 1 & Singapore & 94.0 \\
2 & Finland & 93.2 \\
3 & USA & 93.1 \\
4 & Korea & 92.3 \\
5 & UK & 88.8 \\
6 & Japan & 88.3 \\
7 & Sweden & 87.8 \\
8 & Denmark & 83.5 \\
9 & Taiwan & 83.5 \\
10 & Netherlands & 82.5 \\
11 & Australia & 82.1 \\
12 & Canada & 81.8 \\
13 & Switzerland & 81.3 \\
14 & Germany & 80.1 \\
15 & Italy & 79.1 \\
\hline
\end{tabular}

Similarly, for individuals, $80 \%$ visited government websites in the past 12 months and over $90 \%$ espoused satisfaction with the ease of locating and comprehending the information provided (Figure 3).

Given the appetite for e-services/platforms and the extraordinary ability of the government to provide them, it is hardly surprising that Singapore ranks highly on cloud readiness - a collection of variables that measure the propensity for cloud adoption. Singapore is ranked ahead of her ASEAN peers in 2011 and inched ahead in 2016 (Figure 4).

The Asian Cloud Computing Association (ACCA ${ }^{1}$ ranked countries in Asia Pacific and Oceania on 10 indicators, which Singapore emerged second behind Hong Kong and ahead of New Zealand (\#3), Australia (\#4) and Japan (\#5) among 14 countries in 2016. Specifically, Singapore scored well in broadband quality, data privacy, government regulation, intellectual property protection, but less well in cybersecurity and freedom of information.

Satisfaction with overall quality of Government e-services (FY11-FY15)

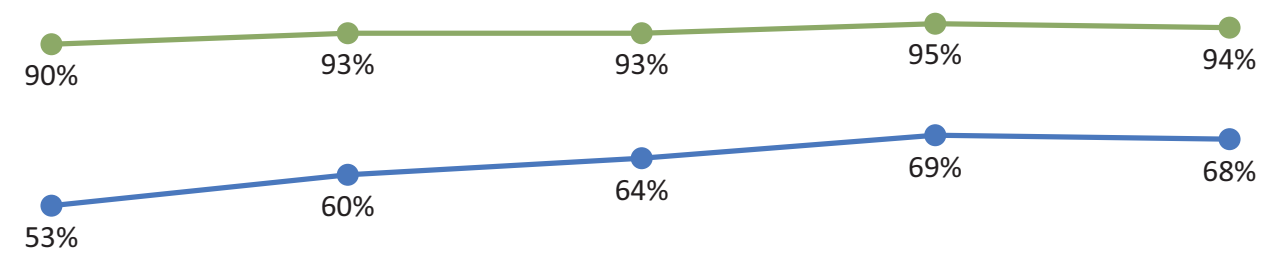

\begin{tabular}{ccccc}
\hline FY11 & FY12 & FY13 & FY14 \\
& - - Satisfied (Rating of 4 and above) & - - Very Satisfied (Rating of 5 and above) &
\end{tabular}

Figure 2. Business users' satisfaction with overall quality of government e-services from 2011-2015.

Satisfaction with overall quality of Government e-services (FY12-FY15)

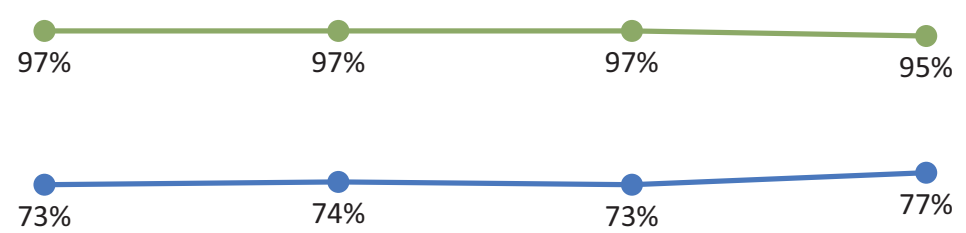

$\begin{array}{ccc}\text { FY12 } & \text { FY13 } & \text { FY14 } \\ & \text { FY15 } \\ - \text { Satisfied (Rating of } 4 \text { and above) } & - \text { Very Satisfied (Rating of } 5 \text { and above) }\end{array}$

Figure 3. Citizens' satisfaction with overall quality of government e-services from 2012-2015.

\footnotetext{
${ }_{1}^{1}$ More information about the methodology and data sources for the index are available at: www.asiacloudcomputing.org/17-news/306-2016-cloudreadiness-index
} 


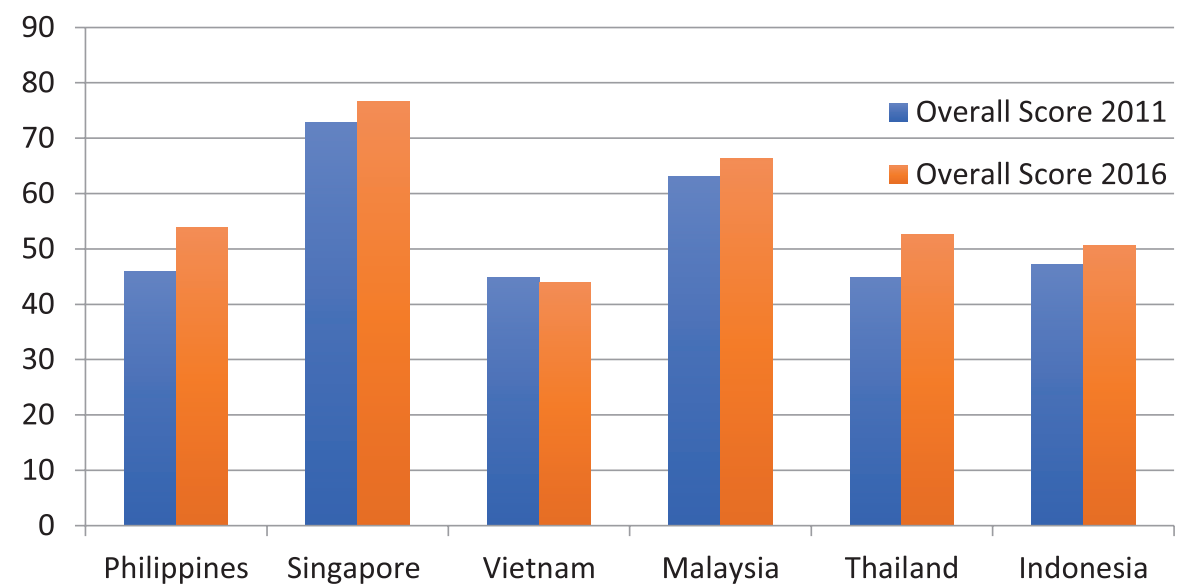

Figure 4. Overall cloud readiness index in ASEAN, 2011, 2016. Source of raw data: Cloud readiness index, 2016.

\subsection{Focus on Whole-of-Government Policies and Practices}

The public service is moving towards a whole-ofgovernment approach to policy making and operations, providing a key driver for whole-of-government cloud adoption. This approach reflects the reality that many contemporary issues cannot be siloed. For example, ageing is not only a health issue, it also impacts the agencies in transport, environment, social and family. To create opportunities for silos to "talk" to each other, the government organised forums for top leaders within different sectors to discuss and craft policies. For example, the Social Forum brings together senior leaders of all the social agencies to build consensus for social policies. This is also great platform to commission important analytics projects that require data from multiple agenciesa typically arduous process. When these projects are debated and benefits delineated among senior leaders across agencies, they are more willing to share data that contribute to a common good. Such initiatives provide use cases for whole-of-government cloud adoption.

Besides platforms to talk between silos, the Prime Minister's Office started an important team of "bridge builders" - The Strategy Group-to shepherd and coax whole-of-government practices. They staff the sectoral forums, and pioneered the data science commissioning platform to nudge line agencies towards a more whole-of-government approach to policy, practice, and data sharing. At the tactical level, Singapore formed a Municipal Services Office whose app is likened to a onestop shop for public feedback and manages it across government to ensure a coordinated response. Such centralisations at the strategic and tactical levels are key drivers for cloud computing adoption in the public service.

\subsection{Restructuring Technology Agencies to Integrate Strategy and Implementation}

To enable whole-of-government innovation and implementation, technology-related agencies are also being re- structured. Telco and media regulation are now brought under one agency-the Info-communications Media Development Authority of Singapore (IMDA). Merging both the InfoComm Development Authority of Singapore (IDA), and the Media Development Authority of Singapore (MDA), IMDA will focus on leveraging new technologies for better regulation and application to improves lives.

Since May 2017, the Prime Minister's Office (PMO) started the Smart Nation and Digital Government Office and assigned a Permanent Secretary to lead this group that integrated strategy and implementation capabilities. The strategic capabilities are integrated from the teams formerly in the PMO, Ministry of Finance, and Ministry of Communication and Information. These teams will be responsible for policy and strategy which will be implemented by Government Technology Agency (GovTech) consisting of deep capabilities in data science and software development.

Other developments include bringing together cybersecurity capabilities through the formation of the Cyber Security Agency (CSA) in 2015 to implement the National Cybersecurity Masterplan 2018. On the local front, the Municipal Services Office (MSO) was established in 2015 to coordinate whole-of-government responses to municipal services that were previously managed by different agencies. These major restructuring exercises are both expensive and expansive but are effective to integrate strategy and implementation.

\subsection{Building the Smart Nation Platform}

A key catalyst of cloud computing is the Smart Nation Platform (SNP) involving a nationwide communications and sensor infrastructure. This platform will enable centralised data aggregation and sharing, as well as provide new capabilities to derive refined insights from cross-referencing a vast array of Government datasets. Datasets can also be shared with the community and industry for co-creation and self-enablement. The SNP will be a key enabler for Singapore to remain on the cutting edge of Government operations and service delivery. 
The SNP will connect sensors through the deployment of Aggregation Gateway Boxes (AG Boxes) throughout Singapore. Approximately 2000 AG Boxes could be deployed at major roadside locations and 10,000 AG Boxes at residential estates. AG Boxes provide connectivity to street lights and wireless mesh networks to ensure that wireless sensors can be easily plugged into the network. As AG Boxes are not licensable telecommunication services, the government will own and operate then. Private operators could share the remaining space in each AG box.

The network will carry sensitive data from sensors so the government's ownership of relevant portions of the SNP will assure that the critical components within the network are treated with the highest security and trust. Specifically, the Info-communication and Media Development Authority will own the infrastructure. In doing so, they are not welded to any contractor and can leverage on different vendors to meet changing demands. Private companies will operate licensed services such as the transmission of data and a majority of maintenance.

Essentially, the SNP is projected to carry a significant amount of Government sensor data. Hence, an integrated platform for the collation, sharing, and analytics will ensure more coherent insights and swifter deployment of government services. The cloud computing backbone is the Smart Nation Operating System (SN-OS) that pull together all sensor and other data types for sensemaking. The SN-OS consists of three platforms: sensor management, data exchange and sense-making.

Through the SN-OS, public sector entities will be able to access cross-agency sensor data to analysis and decrease duplicity of data collection. An analytics layer will facilitate the merging of different datasets (e.g., sensor and admin) to inform policy positions and research. Underlying all this cloud infrastructure is a data governance framework with proper access restrictions and audit trails to ensure that that only the right officers can access the data.

In sum, the Smart Nation Platform is an ambitious example of a comprehensive and massive cloud computing platform that sets the foundation for whole-ofgovernment policy-making and practices.

\subsection{Purpose-Driven Cloud Applications in Healthcare}

\subsubsection{Healthcare Transformation}

Within the healthcare space, cloud computing is applied at scale to healthcare transformation. Signalling the government's commitment to transformation, the Ministry of Health $(\mathrm{MOH})$ recently appointed outgoing National University of Singapore President and former $\mathrm{MOH}$ Director of Medical Services, Professor Tan Chorh Chuan, to direct the inaugural Healthcare Transformation Office as Singapore's first Chief Health Scientist.

Setting up this important national function will accelerate the use of cloud computing especially in popula- tion health analytics and tele-medicine. Nationally, the healthcare sector was restructured into three clusters where each cluster consists of entities in the continuum of care (e.g., acute, community, primary care and preventative health). Each cluster is responsible not only for acute care through large tertiary hospitals but also population health within the geographic cluster. This involves disease prevention, acute care, chronic disease management, and long-term care.

Each cluster utilises their own cloud which is linked to the common registry, a large private cloud known as the National Electronic Health Records (NEHR). This national database contains demographics, subvention and financial data that can be used to stratify the population according to risk for various health conditions and frequent admission ( $\mathrm{Ng}$, Hiew, Goh, \& Tan, 2018). New data sources from other government agencies such as social and family relationship, birth and death data are explored to further enrich the registry. Further, the registry is enriched by longitudinal and multi-disciplinary data around each individual's health.

Such a cloud platform yields invaluable insights on the ageing population that will support primary prevention efforts to better manage the size of population with chronic diseases. At the national level, disability projections (e.g., Ng, Lim, Saw, Francis-Tan, \& Tan, 2018) are done, along with predictive modelling to stratify the population and predict the propensity of groups to develop pre-identified medical conditions such as heart diseases and diabetes.

\subsubsection{Tele-Medicine}

Another application of cloud computing is tele-medicine. The aim of tele-health is to shift from institution-based care towards home and community care, augmenting healthcare resources (e.g., allied health professionals, mental health psychiatrists etc.) in the system. Essentially such technologies shift from a doctor-centric model to a team-based model to manage the shortage of doctors and promote holistic care.

Tele-health applications bring cost-effective supervised rehabilitation to older homebound patients who face significant challenges of traveling to outpatient rehabilitation centers. By exploiting the latest technologies to develop rehabilitation devices specially designed for home and remote use, homebound patients do not need to travel to outpatient rehabilitation centres. Such videoconferencing technology will also help patients to seek advice and guidance from all members of the multidisciplinary rehabilitation team. Tele-rehabilitation will be cost-effective as the rehabilitation team will not need to travel to homebound elderly homes.

Of broader significance, tele-medicine creates a platform for the transmission of real-time data. This has two benefits. One, the rehab team could monitor patients more intensively and calibrate the rehab exercise more appropriately. Two, with cloud computing enabled 
tele-monitoring, care-givers can remotely track elderly citizens or immobile individuals living alone through home/wearable sensors to detect and respond early to unusual extended periods of non-movement or potential falls. Consumers can utilise Telehealth services from their home/ community for greater access to health education and to obtain timely proper care, which has been previously confined to specialised healthcare settings.

The former Info-communication Development Authority of Singapore (IDA) is rolling out the infrastructure for nationwide ultra-high-speed broadband access of $1 \mathrm{Gbps}$ and more, known as the Next Generation Nationwide Broadband Network, to all physical addresses including homes, schools, government buildings, businesses and hospitals. Such infrastructure will support the implementation of a nation-wide tele-rehabilitation program for patients.

Another cloud computing application is a commercial app to link patients and doctors by a Singapore-based company, RingMD that manages 1.5 million patients in over 50 countries. The firm aims to provide healthcare to under-served population by accessing the best doctors through tele-consultations. This is predicated on high mobile phone penetration rates in both developed and developing countries, enabling these consultations through mobile phones and devices.

Users enrol on the RingMD platform and consultations are done through a video link on mobile devices. Individuals can also wear devices that transmits their vital signs to doctors' in real time. Conditions that do

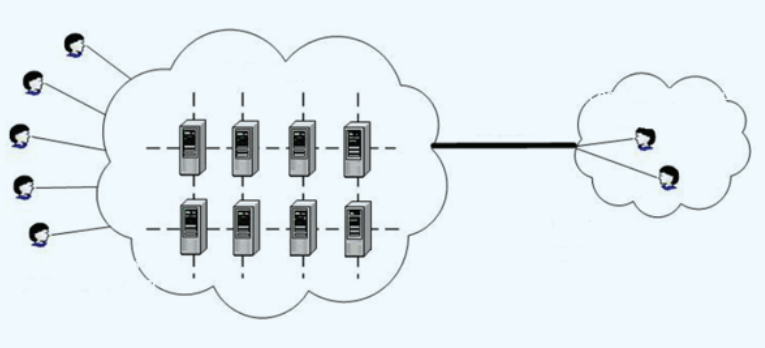

Public cloud. The cloud infrastructure is assessed by the general public or a large industry group.

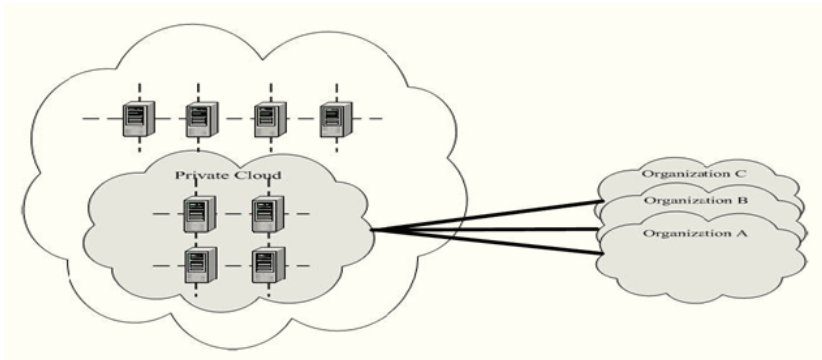

Community cloud. The cloud infrastructure is shared by several firms within a sector with shared considerations (e.g., security, compliance, etc). not require physical checks can be remotely diagnosed and treated. Through analysing copious amounts of data, global and local insights can be provided to patients, doctors and caregivers.

In sum, the key driver for cloud computing in Singapore is purpose-driven, especially in healthcare through healthcare transformation and tele-medicine. With the formation of a new healthcare transformation office driving population health analytics, and a bustling commercial health sector, the adoption of cloud computing is projected to accelerate.

\section{Cybersecurity and Cultural Considerations in Singapore's Cloud Adoption}

There are four models of cloud adoption (Figure 5): public cloud, as the name suggests, is accessed by the general public. An example is the government sharing portal data.gov.sg where government datasets are shared for cocreation and public consumption. The use of public cloud benefits from the lower computing cost. Community Cloud serves a sector and is best exemplified by the Singapore Ministry of Education's iCONnect system, an e-mail and collaboration platform for the teaching profession. Both the public and community clouds are within the medium assurance zones where computing resources are shared with different cloud users at decreased cost.

A private cloud is used for most of government agencies. Known as the Central G-Cloud, it meets high assurance needs of providing a dedicated computing resource

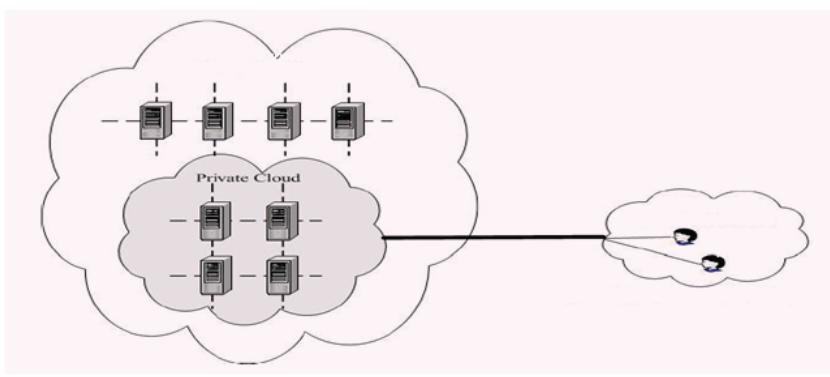

Private cloud. The cloud infrastructure is operated solely for an organization.

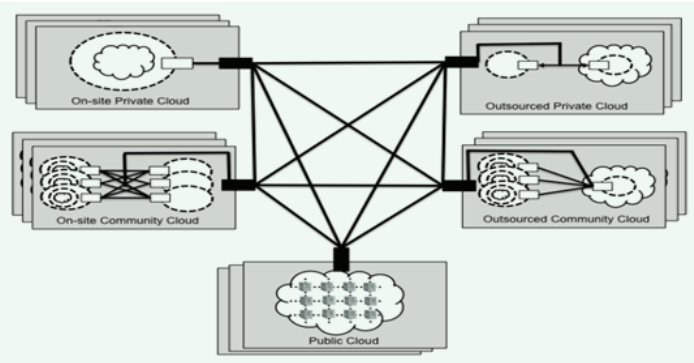

Hybrid cloud. The cloud infrastructure is a composition of two or more clouds (private, community or public). Though these respective clouds have unique properties, they have common applications.

Figure 5. Four models of cloud adoption (Ernst \& Young Global Limited, 2014). 
within government. Most web service exchange and gateways leverage the G-Cloud. The predominant use of a private cloud is driven, among other reasons, by cybersecurity and a prevention-focused cultural tendency.

Cross-cultural experts who categorise national cultures have often described Singapore as "tight", characterised by strong social norms and low patience for deviant behaviour. In a study of 33 countries, Singapore emerged in the top quartile of tightness scores. In other studies, Singapore was categorised as a preventionfocused society where civil servants are detail-orientated and place great emphasis on the absence of negative outcomes, perpetuating a state of vigilance where individuals are careful not to make mistakes (Brown, Abdallah, $\& \mathrm{Ng}, 2011$; Higgins et al., 2001), and high on pettiness (Ng \& Levy, 2018). The opposite is that of a promotionfocused society where the focus is on nurturance, and the presence of positive outcomes (Hamamura, Meijer, Heine, Kamaya, \& Hori, 2009). These empirical studies on culture provide an indication of the mindset of the government in employing a private cloud.

More importantly, a private cloud attempts to mitigate cyber-security risks. As seen in Figure 6, Singapore's cyber-security prowess is not as strong compared with Australia, South Korea and India according to the Cloud Readiness Index 2016 compiled by the ACCA. In new world of data explosions, information security is of paramount consideration. There is intense competition between cryptographers attempting to protect data and hackers attempting to steal it. When the latter happens, public trust is undermined. With Singapore's push to build the world's first Smart Nation, an unprecedented number of devices are being connected to the internet, escalating the risk of expansive disruptions in cyberspace, potentially disrupting defence, public transport and stock markets.
Exacerbated by the Internet of Things, the Cambridge Analytica scandal (Ng, 2018), the accessibility and decreasing cost of hacking tools, have brought about increased risks, enabling lone-wolfs and terrorists to wreck cyber havoc. Over $5 \%$ of American organisations lost north of US\$1 million in 2013 to cyber-crime. More staggering, managing the fallout from hacks costs the world about US\$445 billion per year. Further, the obscure origins of cybercrime make it difficult to differentiate between state and non-state actors. The challenges that cyber-enabled threats bring for Singapore will be non-trivial.

Against the background, Singapore embarked on a coordinated approach to deal with cyber-enabled threats at the government level. Cyber security is transiting from prevention to response and recovery, with the not-if-butwhen mindset. Infrastructure development and policy planning also consider resilience and incident-response capabilities. This is exceptionally paramount in critical information infrastructure (CII) sectors such as banking, transport, health, energy, and telecommunications.

Taken together, the increasing barrage and sophistication of cyber-attacks, a prevention-focused mindset, and the imperative to protect against more damaging cyber-attacks have pushed the government to adopt a private G-Cloud. Though the costs are significantly higher, the Singapore government has probably calculated that the benefits outweigh the cost; more specifically - the potential cost of not implementing a private G-Cloud outweigh the actual cost of doing so.

\section{Recommendations for Cloud Computing Adoption in Big Data Applications}

Learning from Singapore's advances in the cloud computing journey, we propose the following recommenda-

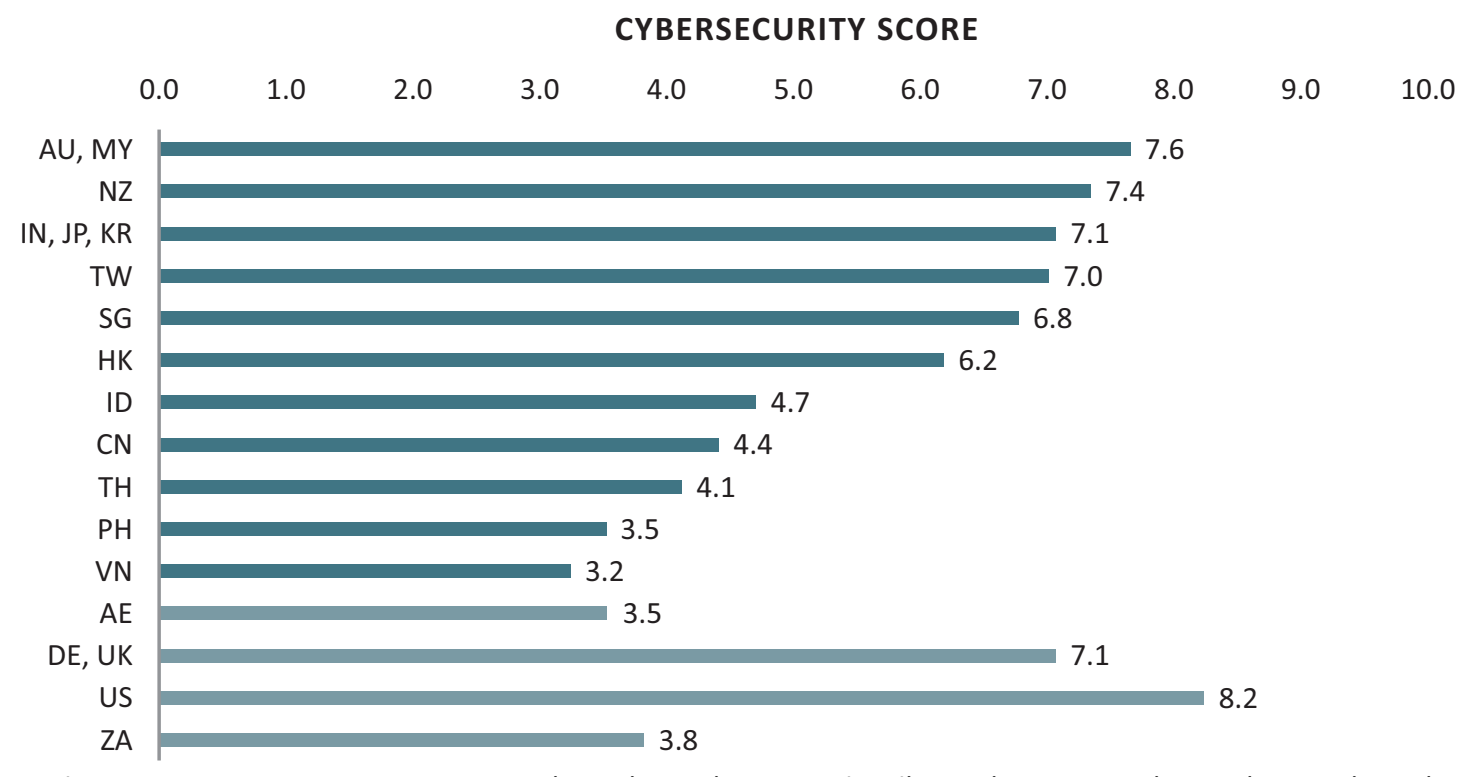

Figure 6. Cybersecurity: Singapore vs. ASEAN (2016). NZ (New Zealand), HK (Hong Kong), TW (Taiwan), JP (Japan), AU (Australia), SG (Singapore), KR (South Korea), PH (Philippines), TH (Thailand), ID (Indonesia), MY (Malaysia), VN (Vietnam). 
tions for cloud computing adoption: Technology, regulation and partnerships. Technologically, embrace cloud analytics, and explore "fog computing"-an emerging technology that enables on-site data sense-making before transmission to the cloud. Promote regulatory sandboxes to experiment with policies that proactively manage novel technologies and business models that may radically change society. Seek out unconventional partnerships, beyond the PPP model, to co-innovate on the initiatives to address perennial issues like the skills-gap.

\subsection{Accelerate Cloud Analytics and Explore Fog Computing}

On the technological front, the current phase for cloud computing is management and organisation with realtime data that are high in volume, velocity and variety. The next phase will be the ability to make sense of the endless streams of data and provide on-demand analytics (Figure 7). While there are fore-runners in the cloud management space, few have been distinguished as market leaders in cloud analytics. Countries can attract upand-coming firms in cloud analytics and invite them to advance their research and operations.

Fog Computing is another technological advancement to explore. Singapore's Smart Nation and other smart city initiatives around the world have led to many Internet-of-Things (IOT) nodes that ingest data but unable to perform analytics. Cloud servers are too far away to analyse data and respond real-time when required. By 2020 , IDC projects that $10 \%$ of the world's data will be produced by these devices at the edge. Fog computing is an edge layer with analytics and artificial intelligence (AI) capabilities. The advantages are analytics-on-site and the

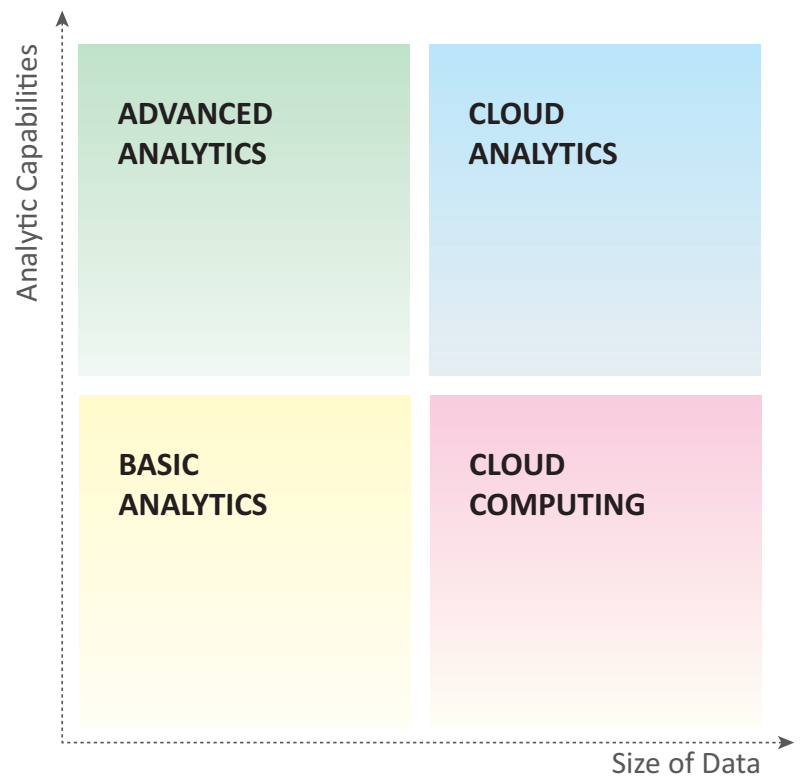

Figure 7. Cloud analytics is a key capability given that data volume and the need for sense-making is increasing exponentially (Hamilton, 2012). transmission of only relevant data to cloud servers, driving down costs.

\subsection{Create Regulatory Sandboxes for Policy Experimentation}

On the regulatory front, the speed of technological advancements has resulted in changes in business models and societal behaviour that are too rapid for policy/regulations to manage. Often, policies and regulations react rather than proactively manage emerging changes, and may hinder the flourishing of new technology. The Monetary Authority of Singapore (MAS), the nation's central bank innovatively created regulatory sandboxes-spaces for policy experimentation-as a means to co-innovate on policies and practices for the explosive FinTech sector. In the sandbox, regulations are relaxed to allow the experimentation of promising fintech products $(\mathrm{Ng}, 2017)$. If the outcomes are successful, both the service and policy innovations can be scaled up and implemented. Such incubation practices can be applied beyond the financial sector for policy innovations in the health, social and transport sectors. Further, public perceptions and stereotypes can be measured at different milestones using computational linguistics (e.g. on age stereotypes; Ng, Allore, Trentalange, \& Levy, 2015) to shape policy communications.

\subsection{Establish Unconventional Partnerships to Co-Innovate on Challenges Like the Skills-Gap}

With the gravitation of tech jobs towards certain talent ultra-hubs, there is an urgent need to develop a base with deep and transferable skills for a city aspiring to be such a hub. The prediction of future skills is challenging; the training of a critical mass on these skills to meet future demand is even more challenging. Institutions need a lead time to design new training course that respond to market demands. When these courses are designed and students enrolled, the demand may have shifted.

Attempting to tackle the skills-gap in Singapore, the Lee Kuan Yew School of Public Policy at the National University of Singapore, signed a Memorandum of Understanding (MOU) with government agencies (SkillsFuture Singapore at the Ministry of Education), Technology firms (Microsoft and Linkedin), and the union (National Trade Union Congress). This MOU seeks to mine public and public-sector data to understand the supply and demand of skills within different occupations. Such insights empower individuals to skill up appropriately, training institutions on the types of courses to offer to meet future demand, and government on programs to meet sectoral demand.

Importantly, this MOU represents an unprecedented multilateral partnership between different parties with unique resources to contribute towards addressing a sticky problem such as the skills-gap. Such a partnership across ASEAN could be leveraged to engender a collab- 
orative approach to address a host of labour and social issues that accompany the fourth industrial revolution.

\section{Conclusion}

This is one of the first known analysis of cloud computing in Singapore with the emphasis of adoption for big data applications in governance and policy. We identified five key drivers: (1) public demand for and satisfaction with e-government services; (2) focus on wholeof-government policies and practices; (3) restructuring of technology agencies to integrate strategy and implementation; (4) building the Smart Nation Platform; (5) purpose driven cloud applications especially in healthcare. These key drivers could serve as learning points and considerations for other nations embarking on their cloud journey.

\section{Conflict of Interests}

The author declares no conflict of interests.

\section{References}

Berman, S., Kesterson-Townes, K., Marshall, A., \& Srivatbsa, R. (2011). The power of cloud: Driving business model innovation. Somers, NY: IBM Institute for Business Value. Retrieved from http://www. ibm.com/cloud-computing/us/en/assets/power-ofcloud-for-bus-model-innovation.pdf

Deeter, B., \& Shen, K. (2015, June 18). The state of the cloud-2015. Bessemer Venture Partners. Retrieved from https://www.bvp.com/blog/state-cloud-2015

Hamilton, B. A. (2012). Cloud analytics playbook. McLean, VA: Booz Allen Hamilton. Retrieved from https://www.slideshare.net/BoozAllen/1111212-cloud -playbook-digital

Brown, J., Abdallah, S. S., \& Ng, R. (2011). Decision making styles East and West: Is it time to move beyond cross-cultural research? International Journal of Sociology and Anthropology, 3, 452-459.

Ernst \& Young Global Limited. (2014). Building trust in the cloud. London: Ernst \& Young Global Limited. Retrieved from http://www.ey.com/Publication/vw LUAssets/EY_-_Building_trust_in_the_cloud/\$FILE/
EY-grc-building-trust-in-the-cloud.pdf

Hamamura, T., Meijer, Z., Heine, S., Kamaya, K., \& Hori, I. (2009). Approach-avoidance motivation and information processing: A cross cultural analysis. Personality and Social Psychology Bulletin, 35, 454-462.

Hashemi, S., Monfaredi, K., \& Masdari, M. (2013). Using cloud computing for e-government: Challenges and benefits. International Journal of Computer, Information, Systems and Control Engineering, 7(9), 596-603.

Higgins, E. T., Friedman, R. S., Harlow, R. E., Idson, L. C., Ayduk, O. N., \& Taylor, A. (2001). Achievement orientations from subjective histories of success: Promotion pride versus prevention pride. European Journal of Social Psychology, 31, 3-23.

$\mathrm{Ng}$, R. (2017, March 29). Learning the art of implementing analytics. Business Times. Retrieved from https://www.businesstimes.com.sg/opinion/learning -the-art-of-implementing-analytics

$\mathrm{Ng}$, R. (2018, June 28). Learning from the Cambridge Analytica fiasco. Business Times. Retrieved from https://www.businesstimes.com.sg/opinion/learning -from-the-cambridge-analytica-fiasco

Ng, R., \& Levy, B. (2018). Pettiness: Conceptualization, measurement and cross-cultural differences. PLOS ONE, 13(1). https://doi.org/10.1371/ journal.pone.0191252

Ng, R., Lim, S.Q., Saw, S.Y., Francis-Tan, C., \& Tan, K.B. (2018). 40-year Projections of disability and living arrangements of older adults in Singapore. Submitted for publication.

Ng, R., Hiew, Y.L., Goh, H.L., \& Tan, K.B. (2018). Implementing a nationwide predictive model for frequent readmissions in Singapore public hospitals. Submitted for publication.

Ng, R., Allore, H., Trentalange, M., Monin, J., \& Levy, B.R. (2015). Increasing Negativity of Age Stereotypes across 200 Years: Evidence from a Database of 400 Million Words. PLOS One, 10(2), e0117086. doi: 10.1371/journal.pone.0117086.

PRWeb. (2012). Garner predicts cloud computing spending to increase by 100 percent in 2016. PRWeb. Retrieved from http://www.prweb.com/releases/ 2012/7/prweb9711167.htm

\section{About the Author}

Reuben Ng spent 16 years in government, consulting, and research. In government, he was in the Prime Minister's Office of Singapore driving evidence-based policy-making through data analytics, and Smart Nation strategies. In consulting, he co-built the Advanced Analytics practice at a top firm and implemented complex analytics projects across industries and functions. In research, he is an expert in quantitative social sciences, social gerontology, and credited with creating innovative techniques to measure societal perceptions/stereotypes that are applied to policy, and program evaluation. Prof. Ng trained as a behavioural scientist at NUS, Oxford and Yale where he was Singapore's first Fulbright Science and Technology Scholar. 